\title{
TDP-43 and HERV-K Envelope-Specific Immunogenic Epitopes Are Recognized in ALS Patients
}

\author{
Elena Rita Simula ${ }^{1}$ (D), Giannina Arru ${ }^{2}$, Ignazio Roberto Zarbo ${ }^{2}$, Paolo Solla ${ }^{2}$ and Leonardo A. Sechi ${ }^{1,3, *(D)}$ \\ 1 Department of Biomedical Sciences, University of Sassari, Viale San Pietro 43b, 07100 Sassari, Italy; \\ simulaelena@gmail.com \\ 2 Department of Clinical, Surgical and Experimental Sciences, University of Sassari, Viale San Pietro 8, \\ 07100 Sassari, Italy; giarru@uniss.it (G.A.); irzarbo@uniss.it (I.R.Z.); psolla@uniss.it (P.S.) \\ 3 Struttura Complessa Microbiologia e Virologia, Azienda Ospedaliera Universitaria, 07100 Sassari, Italy \\ * Correspondence: sechila@uniss.it
}

Citation: Simula, E.R.; Arru, G.;

Zarbo, I.R.; Solla, P.; Sechi, L.A.

TDP-43 and HERV-K

Envelope-Specific Immunogenic Epitopes Are Recognized in ALS Patients. Viruses 2021, 13, 2301. https://doi.org/10.3390/

v13112301

Academic Editor: Craig McCormick

Received: 13 October 2021

Accepted: 15 November 2021

Published: 18 November 2021

Publisher's Note: MDPI stays neutral with regard to jurisdictional claims in published maps and institutional affiliations.

Copyright: (C) 2021 by the authors Licensee MDPI, Basel, Switzerland. This article is an open access article distributed under the terms and conditions of the Creative Commons Attribution (CC BY) license (https:// creativecommons.org/licenses/by/ $4.0 /)$.

\begin{abstract}
The human endogenous retrovirus-K (HERV-K) and TAR DNA-binding protein 43 (TDP43) have been associated with the pathophysiology of amyotrophic lateral sclerosis (ALS). Given these findings, we investigated the humoral response against HERV-K envelope surface (env-su) glycoprotein antigens and TDP-43 in the plasma of ALS patients and healthy controls (HCs). The measured levels of Abs against the different epitopes' fragments were significantly elevated in ALS patients, both in long-survivor (LS) and newly diagnosed (ND) patients, compared to HCs. We observed a positive correlation between HERV-K and TDP-43 antibodies (Abs) levels, which seemed to strengthen with disease progression, that was not found in HCs. The TDP-43 and HERV-K epitopes identified in this study are highly immunogenic and recognized by the humoral response of ALS patients. Increased circulating levels of Abs directed against specific HERV-K- and TDP-43-derived epitopes could serve as possible biomarkers.
\end{abstract}

Keywords: ALS; amyotrophic lateral sclerosis; TDP-43; HERV-K; HML-2

\section{Introduction}

Amyotrophic lateral sclerosis (ALS) is a neurodegenerative disease of unknown etiology described for the first time in 1874 by Jean-Martin Charcot. It is characterized by the progressive degeneration of both the upper and lower motor neurons, which display cytoplasmatic inclusions [1,2]. Population-based studies have highlighted that women are less affected by sporadic ALS (sALS) than men, while the same incidence between men and women in familial ALS (fALS) is observed. The incidence decreases rapidly after 80 years of age, whereas the 58-63 and 47-52 age brackets represent the peak ages of onset for sALS and fALS, respectively [3]. The ALS condition is primarily characterized by the involvement of the pyramidal tract with the progressive reduction in upper motor neuron activity, which originates from the motor cortex, and lower motor neurons, which connect the spinal cord and brainstem to skeletal muscles. Common features of ALS are progressive muscle weakness and atrophy, fasciculations, dysarthria, and dysphagia. Remarkably, a significant proportion of cases presents frontotemporal lobe degeneration (FTLD) with cognitive damage. Respiratory complications are the final stage, after which death occurs within two to five years of diagnosis [4]. The main causes of ALS are still unknown, but significant advances have been made in understanding the environmental and genetic components involved in the pathology [5]. There are several hypotheses behind the development of the disease, involving both genetic predisposition, with more than 24 implicated genes, such as Superoxide dismutase 1 (SOD1) and TAR DNA-binding protein 43 (TDP-43) [6], and environmental risk factors, for instance, smoking [7], physical activity [8], and chemical exposure $[9,10]$. 
Emerging evidence has indicated that human endogenous retrovirus K (HERV-K) may play a role in the disease's etiopathogenesis. Human endogenous retroviruses (HERVs), considered until recently as junk DNA, constitute nearly $8 \%$ of the human genome. Proviral genome integrations into the DNA are remnants of infections that occurred over several million years ago. Nevertheless, numerous nonsense mutations have contributed to their defection [11].

HERV-K, in particular the HML-2 subgroup, is present in hundreds of copies in the human genome and is one of the most recently acquired and the most transcriptionally active among HERVs. The involvement of HERV-K in the pathophysiology of ALS has been documented by numerous groups. Douville et al., (2011) observed an altered expression of HERV-K pol transcripts in post-mortem brain samples of patients affected by ALS compared to other pathological conditions such as Parkinson's disease, chronic systemic illnesses, and healthy subjects [12]. Transgenic animals, in whose neurons the env gene was expressed, developed progressive motor dysfunction [13].

It is noteworthy that HERV-K expression is regulated by TDP-43, which can bind to the long terminal repeat region (LTR) of the virus. Human neurons transfected with TDP-43 showed an increase in HERV-K expression, while knockdown of endogenous TDP-43 resulted in a decrease in HERV-K expression [13]. Furthermore, it was demonstrated that Abs levels against HERV-K-env-su $(20-38)$ were significantly elevated in ALS patients compared to multiple sclerosis (SM), Alzheimer's disease (AD), and healthy control subjects, both in serum and cerebrospinal fluid (CSF), suggesting HERV-K-env-su ${ }_{(20-38)}$ as a possible early novel biomarker [14]. Specific post-translational modifications of TDP-43 may have an impact on HERV-K expression patterns. Concerning this, the formation of TDP-43 aggregates alters HERV-K RT, polyprotein levels, and the cellular localization of the viral proteins [15]. Li et al. [13] demonstrated that HERV-K LTR has four binding sites for TDP43 that have been shown to regulate its activation. In HIV patients an increased nuclear TDP-43 expression accompanied by an enhanced TDP-43 phosphorylation compared to the levels observed in controls has been reported. The co-expression of HERV-K RT and TDP-43 proteins has been observed in the majority of neurons with a significant positive correlation between them [16].

TDP-43 is a multifunctional protein associated with several biological functions [17], including mRNA transcription, splicing and stability [18-20], stress granule formation [21], and Protein-Protein Interactions [22]. TDP-43 is indispensable for the development of the central nervous system (CNS) from the earliest stages of embryonic life to adulthood [23,24]. Under physiological conditions, TDP-43 is mainly a nuclear protein, but it also shuttles to the cytoplasm for further functions. Neurons transfected with TDP-43 mutants altering nuclear trafficking exhibit cytoplasmic aggregates with phosphorylated and ubiquitinated TDP-43, which is characteristic of ALS pathology [25]. The TARDBP gene is located on chromosome 1, and the TDP- 43 protein consists of 414 amino acids. The C-terminal region, which plays a crucial role in the disease, encompasses a prion-like glutamine/asparaginerich $(\mathrm{Q} / \mathrm{N})$ domain and a glycine-rich region [26]. TDP-43 represents a target for numerous post-translational modifications that may change its structure, functions, localization, and its aggregative predisposition $[27,28]$. The most documented post-translational modifications in ALS patients are phosphorylation in serine-409 and serine- $410[29,30]$, but other phosphorylated sites of pathological TDP-43 have also been described, such as serine-379, 403 , and 404 [29,31,32]. Kametani et al., showed that all serine and threonine residues in the C-terminal domain can become phosphorylated [33], and this may increase the tendency of TDP-43 to be hydrolyzed into C-terminal fragments or to aggregate [34].

To date, the ALS diagnostic process requires approximately one year, since only the progression of symptoms and the presence of signs of both upper and lower motor neuron involvement can confirm the ALS diagnosis. The diagnosis is based on clinical examination, electrophysiological findings, medical history, and exclusion of confounding disorders [35]. Unfortunately, no effective therapies able to cure the disease are available; Riluzole is the only therapy that can prolong ALS survival (by approximately three months). Different 
treatments are available to help control ALS symptoms, prevent unnecessary complications, and make it easier for patients to live with the disease. This study aims to more deeply understand the possible relationship between TDP-43 and HERV-K through the investigation of the humoral response to different epitopes of the TDP-43 C-termina region and the HERV-K envelope. The detection of circulating autoantibodies is crucial in the diagnosis and monitoring of several diseases, and it helps to understand the etiopathogenesis. Furthermore, this approach may be useful to hypothesize new biomarkers for ALS which would allow a faster diagnosis.

\section{Materials and Methods}

\subsection{Samples Collection}

We evaluated an ALS group of 45 patients (17 females and 28 males; mean age \pm SD: $64.67 \pm 9.11$ years), and an age- and gender-matched healthy control group (HCs) collected from the Blood Transfusion Centre of Sassari (17 females and 28 males; mean age \pm SD: $63.87 \pm 4.82$ years). Data are summarized in Table 1.

Table 1. Demographic data of investigated groups.

\begin{tabular}{ccccc}
\hline Study Population & & Female & Male & Mean Age \pm SD \\
\hline Healthy controls & & 17 & 28 & $63.87 \pm 4.82$ \\
ALS patients & ND & 7 & 20 & $64 \pm 7.8$ \\
& LS & 10 & 8 & $66.1 \pm 10.9$ \\
\hline
\end{tabular}

The ALS population, sampled from January 2016 to December 2019, comprised newly diagnosed (ND) ALS patients (7 females and 20 males; mean age \pm SD: $64 \pm 7.8$ years), hospitalized at the Neurology Unit Clinic of the University Hospital of Sassari and longsurvivor (LS) ALS patients (10 females and 8 males; mean age \pm SD: $65.8 \pm 10.4$ years) reported by primary doctors and doctors in the Sassari local district.

Peripheral venous blood samples were collected in a K2-EDTA tube. Whole blood was gently layered over an equal volume of Ficoll (Sigma-Aldrich, St. Louis, MO, USA) in a $15 \mathrm{~mL}$ tube and centrifuged for $20 \mathrm{~min}$ at $1800 \mathrm{RPM}$ without brake. The plasma contained in the uppermost layer was collected by pipetting and tested for the presence of Abs against TDP-43- and HERV-K-env-derived epitopes. All subjects gave their informed consent for inclusion before they participated in the study. The study was conducted in accordance with the Declaration of Helsinki, and the protocol was approved by the Ethics Committee of ALS 1 Sassari (2149/CE).

\subsection{Peptides}

The peptides HERV-K-env-su ${ }_{(20-38)}$ (derived from HERV-K env surface protein, UniprotKB, accession number: O42043), TDP-43 ${ }_{(258-271)}, \mathrm{TDP}_{4} 43_{(398-411)}$, and TDP-43 ${ }_{(398-411)} \mathrm{P}$ (derived from TDP-43 protein, UniprotKB, accession number: Q13148) were designed using the Immune Epitope Database and analysis resource (IEDB) and synthesized at $>95 \%$ purity (LifeTein, South Plainfield, NJ 07080, USA). The IEDB software predicts regions of proteins that are likely to be recognized as epitopes in the context of a B-cell response. All peptides were dissolved in DMSO and stored at $-80^{\circ} \mathrm{C}$ in single-use aliquots $(10 \mathrm{mM})$ (Table 2).

Table 2. Epitopes identified in HERV-K-env-su and TDP-43.

\begin{tabular}{ccc}
\hline & Epitope Position & Epitope Sequence \\
\hline HERV-K-env-su $_{(20-38)}$ & aa $20-38$ & VWVPGPTDDRCPAKPEEEG \\
TDP-43 ${ }_{(258-271)}$ & aa $258-271$ & SNAEPKHNSNRQLE \\
TDP-43 ${ }_{(398-411)}$ & aa 398-411 & NGGFGSSMDSKSSG \\
TDP-43 $_{(398-411)} \mathrm{P}$ & aa 398-411 & NGGFGSSMDSK-(PSer)-(PSer)-G \\
\hline
\end{tabular}




\subsection{Enzyme-Linked Immunosorbent Assay (ELISA)}

Indirect ELISA was performed to detect specific Abs against HERV-K-env-su ${ }_{(20-38)}$, TDP-43(258-271), TDP-43(398-411), and TDP-43(398-411) P epitopes. Ninety-six-well Nunc immuno-plates were incubated overnight at $4{ }^{\circ} \mathrm{C}$ with a solution $0.05 \mathrm{M}$ of carbonatebicarbonate, pH 9.5 (Sigma-Aldrich, St. Louis, MO, USA) and the respective peptides at $10 \mu \mathrm{g} / \mathrm{mL}$. The plates were incubated for $1 \mathrm{~h}$ at room temperature in a blocking solution with 5\% non-fat dried milk (Sigma-Aldrich, St. Louis, MO, USA) and phosphate-buffered saline (PBS) and washed twice in a solution with $0.05 \%$ Tween-20 and PBS (PBS-T). The plasma samples were added at 1:100 concentration and incubated for $2 \mathrm{~h}$. After this, each plate was washed five times in PBS-T and incubated for $1 \mathrm{~h}$ at room temperature (RT) with $100 \mu \mathrm{L}$ of PBS and alkaline phosphate-conjugated goat anti-human IgG polyclonal antibody (1:1000, Sigma-Aldrich, St. Louis, MO, USA). After another washing step in PBS-T, plates were incubated in a dark environment for 8 to $10 \mathrm{~min}$ in milli-Q water and p-nitrophenyl phosphate (Sigma-Aldrich, St. Louis, MO, USA), and an absorbance of $405 \mathrm{~nm}$ was recorded using a SpectraMax Plus 384 microplate reader (Molecular Devices, Sunnyvale, CA, USA). Each sample was run in duplicate, and normalization was performed with the positive control included in each assay. Background activity was calculated as the mean signal of an immobilized peptide with secondary Ab. Results are expressed as the means of duplicates of $405 \mathrm{~nm}$ optical density (OD) values.

\subsection{Statistical Analysis}

All data were analyzed using GraphPad Prism 8.2.0 software (GraphPad Software, San Diego, CA, USA). A Mann-Whitney U test was used to analyze non-parametric data and compare differences among two groups. Kruskal-Wallis and Dunn's post hoc tests were carried out to compare differences among three groups. Statistically significant difference was set with a $p<0.05$. Receiver-operating characteristic (ROC) was used to choose the cut-off value to assess the sample positivity, which was consequently tested through Fisher's exact test. A Spearman correlation test was performed among levels of antibodies to HERV-K env- and TDP-43-derived peptides.

\section{Results}

We enrolled 45 ALS patients and 45 matched HCs in our study. Details of the ALS patients' clinical information are shown in Table 3.

The humoral response against HERV-K-env-su $\mathrm{s}_{(20-38)}$ and three selected epitopes of TDP-43 was evaluated in the plasma of the ALS and HCs groups. A Mann-Whitney U test was performed to compare quantitative values of the antibodies' OD among groups. Cutoff values were selected by ROC analysis, and the comparison between the percentage of positive and negative samples was performed using Fisher's exact test. Briefly, Abs against HERV-K-env-su (20-38) were present in $40 \%$ of the ALS population, versus $8.89 \%$ in the healthy control group (Figure 1A): Mann-Whitney $\mathrm{U}$ test, $p<0.0001$, HCs median $=0.139$, $95 \%$ CI [0.107, 0.175], ALS median $=0.371,95 \%$ CI [0.305, 0.45]; cut off value of 0.393 ; Fisher's exact test, $p=0.0011$; and AUC $=0.86$. We also observed a remarkable Abs response against TDP-43 epitopes in the Sardinian population. In particular, $24.44 \%$ of ALS patients showed Abs against TDP-43(258-271), whereas only $4.44 \%$ in the healthy group showed a humoral response against the same peptide (Figure 1B): Mann-Whitney U test, $p<0.0001$, HCs median $=0.157,95 \%$ CI $[0.084,0.191]$, ALS median $=0.284,95 \%$ CI [0.243, 0.318]; cut off value of 0.381; Fisher's exact test, $p=0.013$; and AUC $=0.813$. The ALS patients showed an increased Abs presence against the TDP-43(398-411) epitope (Figure 1C), with a positivity measured in $51.11 \%$ of the ALS group versus $4.44 \%$ of HCs with a Fisher's exact test $p<0.0001$ (Mann-Whitney U test, $p<0.0001$, HCs median $=0.11,95 \%$ CI [0.074, $0.168]$, ALS median $=0.386,95 \%$ CI [0.332, 0.453]; cut off value of 0.385 ; and AUC $=0.915$ ). Figure 1D shows the antibody response against TDP- $43_{(398-411)} \mathrm{P}$; only $4.44 \%$ of healthy subjects were positive, whereas in ALS patients, we observed an increased percentage of up to $20.00 \%$ (Mann-Whitney U test, $p=0.0006$, HCs median $=0.151,95 \%$ CI [0.103, 0.195], 
ALS median $=0.226,95 \%$ CI $[0.202,0.255]$; cut off value of 0.35 ; Fisher's exact test, $p=0.049$; and $\mathrm{AUC}=0.706$ ).

Table 3. ALS patients' clinical data.

\begin{tabular}{|c|c|c|c|c|c|}
\hline ID Patients & Gender & Y/o & Bulbar/Spinal Onset & Disease Duration (Months) & ND-ALS or LS-ALS \\
\hline ALS 1 & M & 74 & bulbar & 1 & ND-ALS \\
\hline ALS 2 & M & 65 & spinal & 1 & ND-ALS \\
\hline ALS 3 & M & 67 & spinal & 1 & ND-ALS \\
\hline ALS 4 & M & 66 & spinal & 1 & ND-ALS \\
\hline ALS 5 & M & 69 & spinal & 23 & ND-ALS \\
\hline ALS 6 & M & 61 & spinal & 7 & ND-ALS \\
\hline ALS 7 & $\mathrm{~F}$ & 58 & spinal & 1 & ND-ALS \\
\hline ALS 8 & M & 66 & spinal & 1 & ND-ALS \\
\hline ALS 9 & $\mathrm{~F}$ & 56 & spinal & 8 & ND-ALS \\
\hline ALS 10 & M & 78 & spinal & 1 & ND-ALS \\
\hline ALS 11 & $\mathrm{~F}$ & 56 & spinal & 8 & ND-ALS \\
\hline ALS 12 & M & 66 & spinal & 14 & ND-ALS \\
\hline ALS 13 & M & 44 & spinal & 16 & ND-ALS \\
\hline ALS 14 & M & 66 & bulbar & 9 & ND-ALS \\
\hline ALS 15 & $\mathrm{~F}$ & 70 & bulbar & 3 & ND-ALS \\
\hline ALS 16 & $\mathrm{~F}$ & 71 & bulbar & 6 & ND-ALS \\
\hline ALS 17 & M & 47 & spinal & 22 & ND-ALS \\
\hline ALS 18 & $\mathrm{~F}$ & 64 & spinal & 23 & ND-ALS \\
\hline ALS 19 & $\mathrm{M}$ & 61 & spinal & 4 & ND-ALS \\
\hline ALS 20 & M & 66 & spinal & 3 & ND-ALS \\
\hline ALS 21 & M & 61 & spinal & 3 & ND-ALS \\
\hline ALS 22 & M & 62 & spinal & 20 & ND-ALS \\
\hline ALS 23 & M & 71 & spinal & 7 & ND-ALS \\
\hline ALS 24 & M & 74 & spinal & 3 & ND-ALS \\
\hline ALS 25 & M & 71 & spinal & 3 & ND-ALS \\
\hline ALS 26 & M & 60 & spinal & 23 & ND-ALS \\
\hline ALS 27 & $\mathrm{~F}$ & 58 & spinal & 22 & ND-ALS \\
\hline ALS 28 & M & 71 & bulbar & 60 & LS-ALS \\
\hline ALS 29 & M & 68 & spinal & 36 & LS-ALS \\
\hline ALS 30 & M & 65 & spinal & 48 & LS-ALS \\
\hline ALS 31 & $\mathrm{~F}$ & 78 & spinal & 24 & LS-ALS \\
\hline ALS 32 & M & 59 & spinal & 240 & LS-ALS \\
\hline ALS 33 & $\mathrm{~F}$ & 76 & bulbar & 132 & LS-ALS \\
\hline ALS 34 & $\mathrm{~F}$ & 81 & bulbar & 48 & LS-ALS \\
\hline ALS 35 & $\mathrm{~F}$ & 74 & spinal & 84 & LS-ALS \\
\hline ALS 36 & $\mathrm{~F}$ & 57 & bulbar & 144 & LS-ALS \\
\hline ALS 37 & M & 58 & spinal & 108 & LS-ALS \\
\hline ALS 38 & $\mathrm{~F}$ & 48 & spinal & 48 & LS-ALS \\
\hline ALS 39 & $\mathrm{~F}$ & 56 & spinal & 72 & LS-ALS \\
\hline ALS 40 & M & 55 & spinal & 72 & LS-ALS \\
\hline ALS 41 & $\mathrm{~F}$ & 65 & spinal & 48 & LS-ALS \\
\hline ALS 42 & $\mathrm{~F}$ & 83 & bulbar & 96 & LS-ALS \\
\hline ALS 43 & M & 82 & bulbar & 96 & LS-ALS \\
\hline ALS 44 & $\mathrm{~F}$ & 55 & spinal & 132 & LS-ALS \\
\hline ALS 45 & M & 58 & spinal & 204 & LS-ALS \\
\hline
\end{tabular}

In order to investigate a potential difference in the humoral response in the plasma of ALS-ND and ALS-LS patients, the Kruskal-Wallis test and Dunn's post hoc analysis were performed. The limitation of this analysis is that the three groups (HCs, ALS-LS, and ALS-ND) are not matched by sex due to the small ALS population; it would be very interesting to more deeply understand if statistical differences correlated with sex exist in a larger population. The obtained results highlight a significant difference in HCs compared to ALS-ND and ALS-LS for the peptides derived from TDP-43 and HERV-K envelope 
protein, while no difference was observed in the human response between ALS-ND and ALS-LS (Figure 2).

A

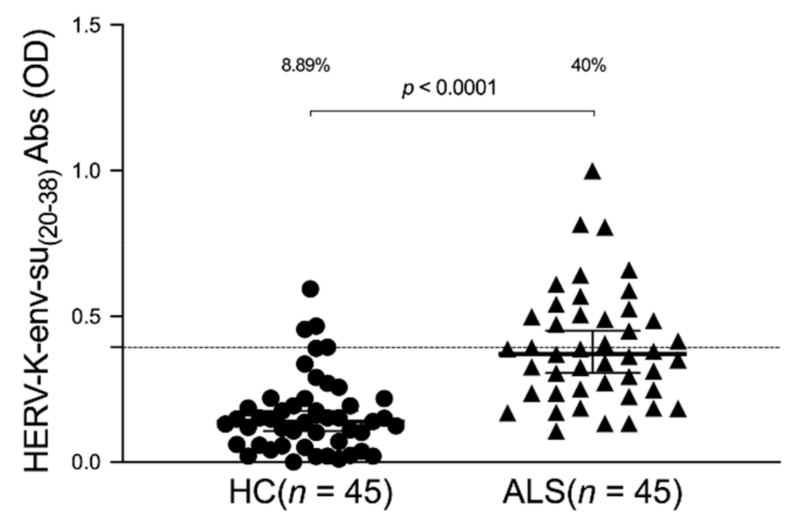

C

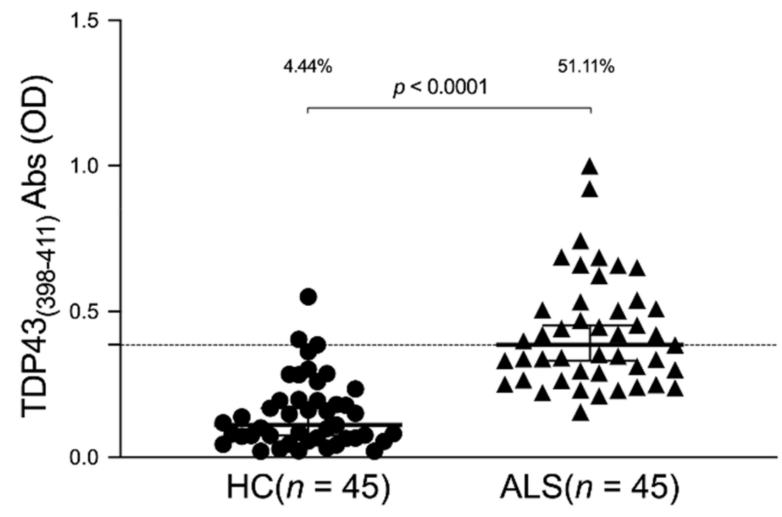

B

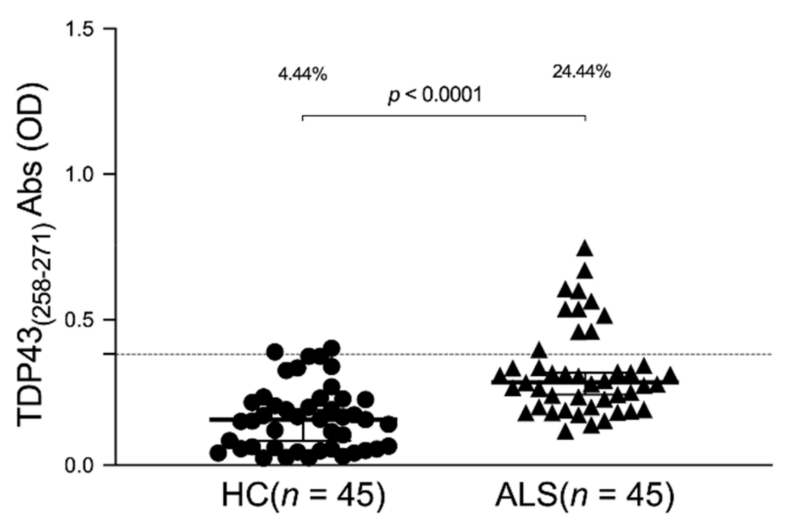

D

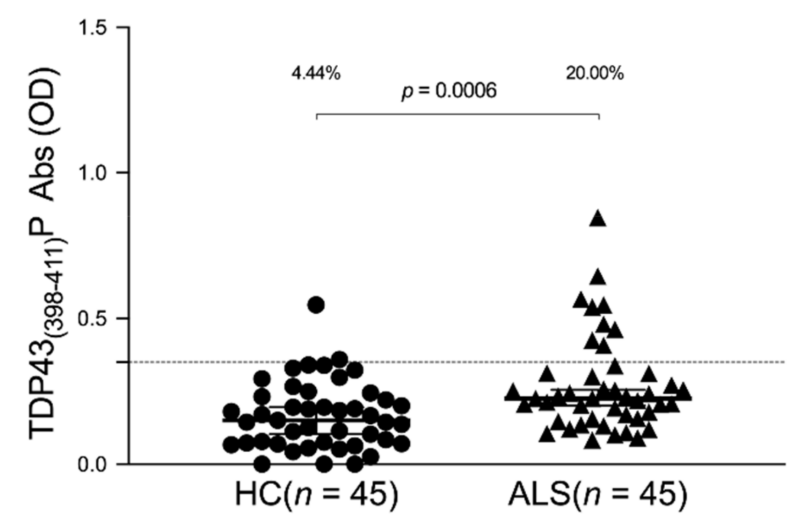

Figure 1. ELISA-based analysis of Abs reactivity against HERV-K- and TDP-43-derived peptides. Plasma samples from ALS patients and HCs subjects were tested against HERV-K-env-su ${ }_{(20-38)}(\mathbf{A}), \mathrm{TDP}_{4} 43_{(258-271)}(\mathbf{B})$, TDP-43(398-411) $($ C), and TDP-43 ${ }_{(398-411)}$ P (D) peptides. Median and dashed lines represent thresholds used to assess the samples' positivity. The Mann-Whitney $p$-value and the percentage of positive patients evaluated by Fisher's exact test are indicated in the upper part of each graph.

Supplementary analysis was performed to evaluate a possible correlation between TDP-43 and HERV-K humoral response. The results show a correlation between the levels of antibodies and HERV-K and TDP-43 epitopes in ALS patients; these correlations are: HERVK-env-su $\mathbf{s}_{(20-38)}$ and TDP-43 ${ }_{(258-271)}(r=0.297, p=0.048)$ (Figure 3A), HERV-K-env-su ${ }_{(20-38)}$, and TDP-43 $3_{(398-411)}(r=0.488, p=0.001)$ (Figure 3C), and finally HERV-K-env-su $(20-38)$ and TDP-43 ${ }_{(398-411)} \mathrm{P}(r=0.435, p=0.003)$ (Figure $\left.3 \mathrm{E}\right)$. The same analysis was performed in HCs to investigate a possible correlation between HERV-K and TDP-43 autoantibody levels. No correlations were observed in HCs; HERV-K-env-su $(20-38)$ and TDP-43 $3_{(258-271)}$ showed $\mathrm{r}$ and $p$ values, respectively, of -0.138 and ns (Figure 3B), while HERV-K-env-su $(20-38)$, and TDP$43_{(398-411)}$ showed an $r=-0.173$ and $p=\mathrm{ns}$, (Figure 3D), and finally HERV-K-env-su (20-38) and TDP-43 ${ }_{(398-411)}$ P showed an $r=-0.099$ and $p=$ ns (Figure 3F). 
A

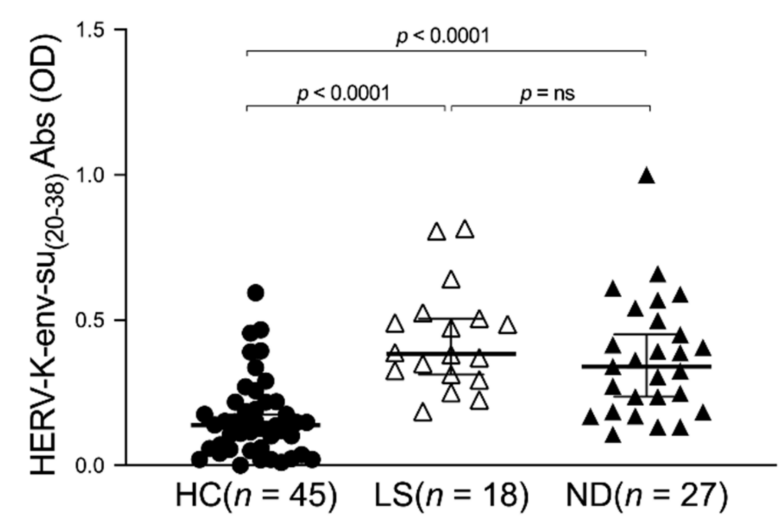

C

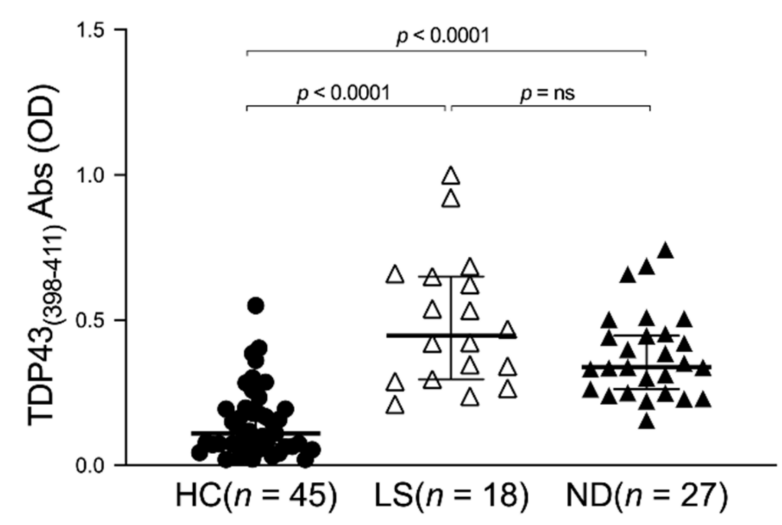

B

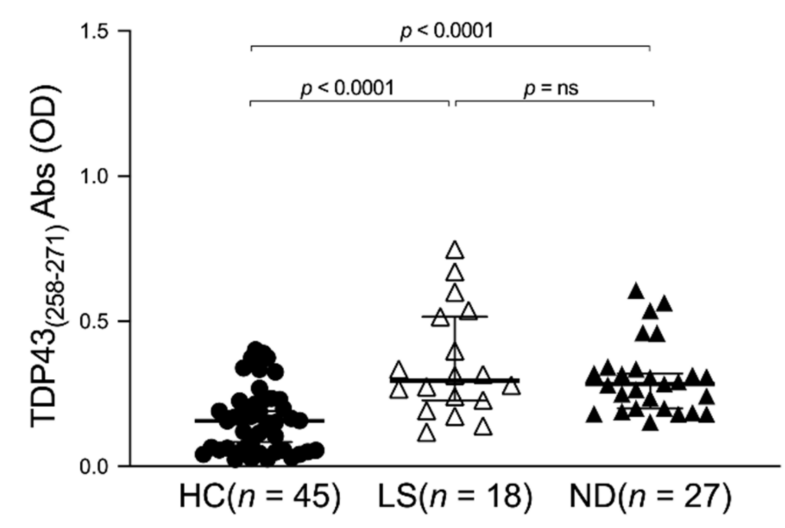

D

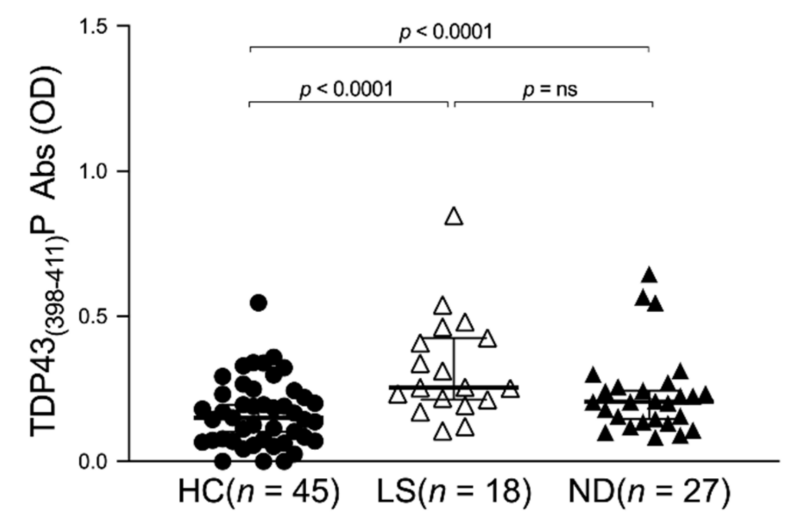

Figure 2. ELISA-based analysis of Abs reactivity against TDP-43- and HERV-K-derived peptides in ALS-ND, ALS-LS, and HCs groups. Plasma samples were tested against HERV-K-env-su (20-38) (A), TDP-43(258-271) $($ B), TDP-43(398-411) $($ C), and TDP-43 ${ }_{(398-411)} \mathrm{P}(\mathrm{D})$ peptides. A Kruskal-Wallis test and Dunn's post hoc analysis were performed. Scatter plots represent the median, and the $p$-value is indicated in the upper part of each graph.

In order to deepen this aspect, we investigated the correlation between the humoral response and TDP-43 and HERV-K in patients divided by ALS-ND and ALS-LS. Briefly, we observed positive correlations between the two epitopes in the ALS-ND group, as follows: HERV-K-env-su ${ }_{(20-38)}$ and TDP-43 ${ }_{(258-271)}(r=0.246, p=n s)$ (Figure 4A), HERV-Kenv-su ${ }_{(20-38)}$ and TDP-43 ${ }_{(398-411)}(r=0.451, p=0.018)$ (Figure $\left.4 \mathrm{C}\right)$, and lastly HERV-K-env$\mathrm{su}_{(20-38)}$ and TDP-43 ${ }_{(398-411)} \mathrm{P}(r=0.357, p=\mathrm{ns})$ (Figure $\left.4 \mathrm{E}\right)$. We observed a positive correlation regarding ALS-LS patients, as follows: HERV-K-env-su $(20-38)$ and TDP-43(258-271) $(r=0.395, p=\mathrm{ns})$ (Figure 4B), HERV-K-env-su ${ }_{(20-38)}$ and TDP-43 ${ }_{(398-411)}(r=0.570, p=0.013)$ (Figure $4 \mathrm{D})$, and finally HERV-K-env-su $(20-38)$ and TDP-43 ${ }_{(398-411)} \mathrm{P}(r=0.473, p=0.047)$ (Figure 4F). 
A

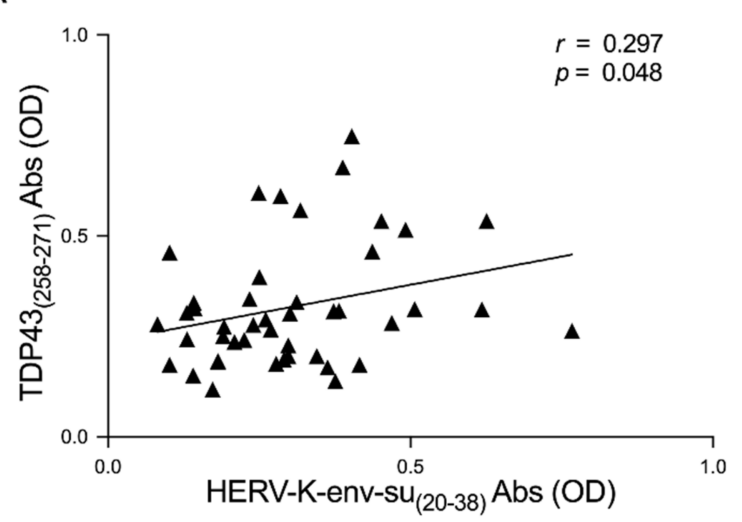

C

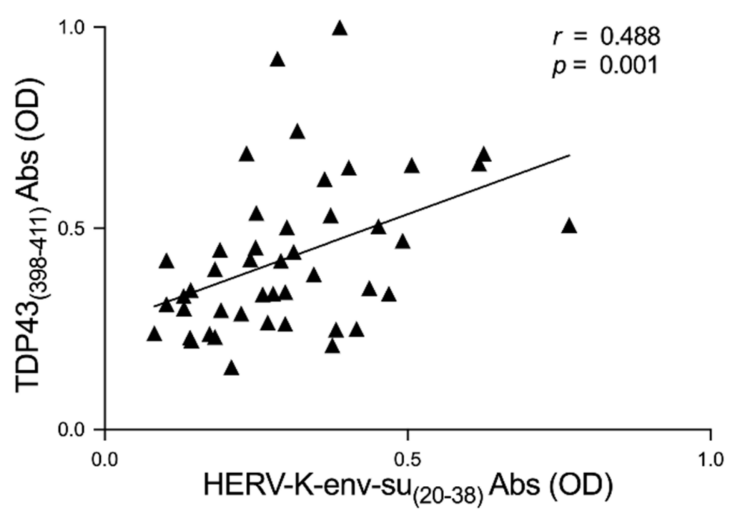

$\mathrm{E}$

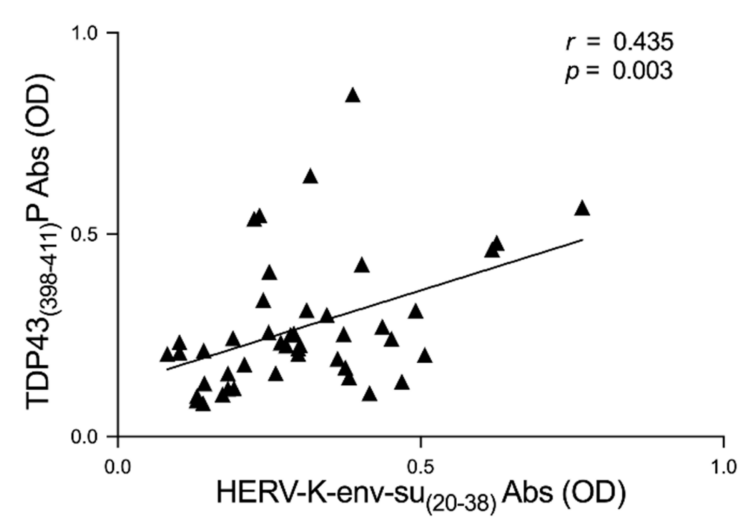

B

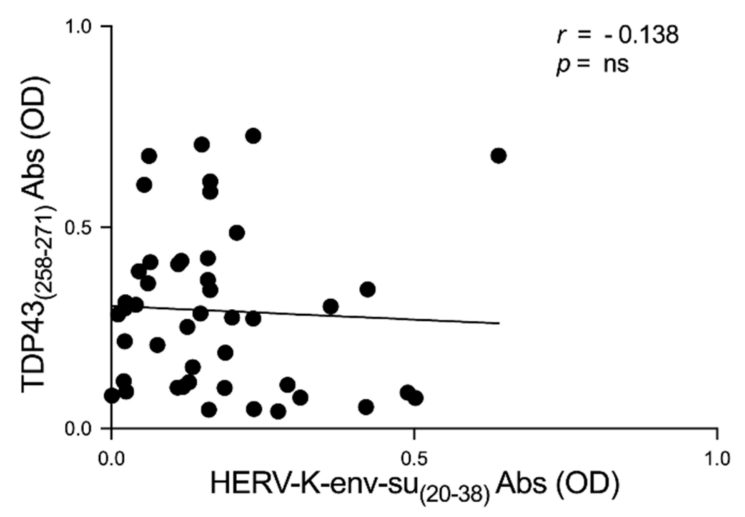

D

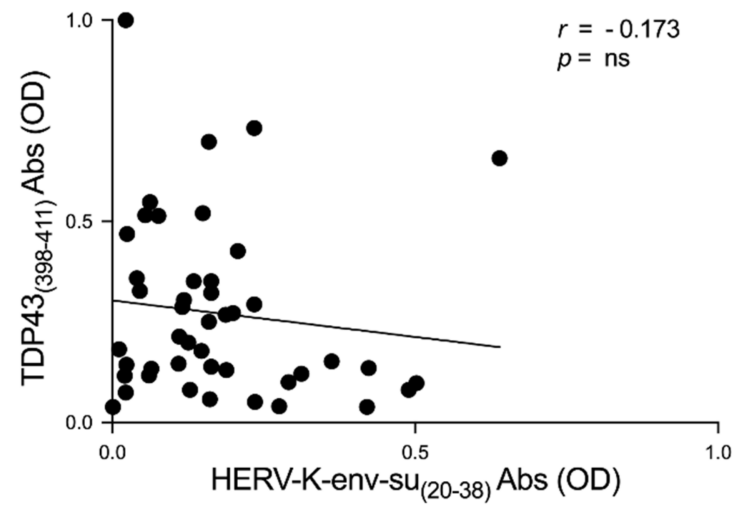

$\mathrm{F}$

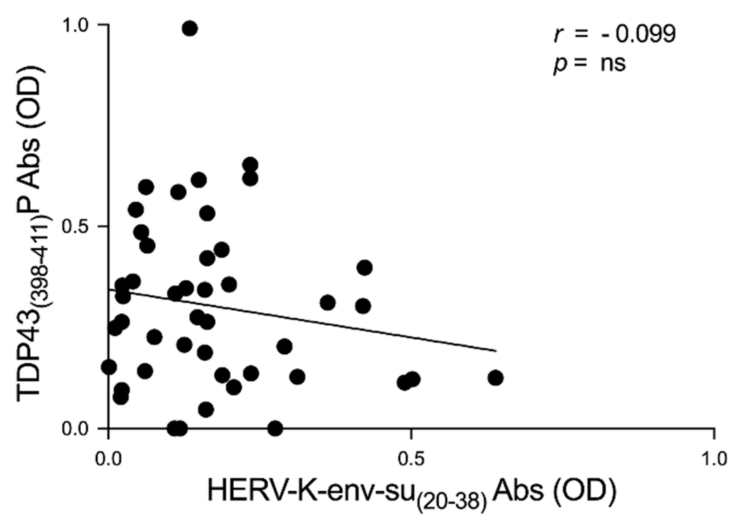

Figure 3. Scatter plots of antibodies to TDP-43, derived peptides, and HERV-K env epitope in ALS populations. The graphs show the correlation between HERV-K-env-su $(20-38)$ and TDP-43 ${ }_{(258-271)}$, TDP-43 $3_{(398-411)}$, and TDP-43(398-411) $\mathrm{P}$ in ALS patients (A,C,E) and in HCs (B,D,F). 
A

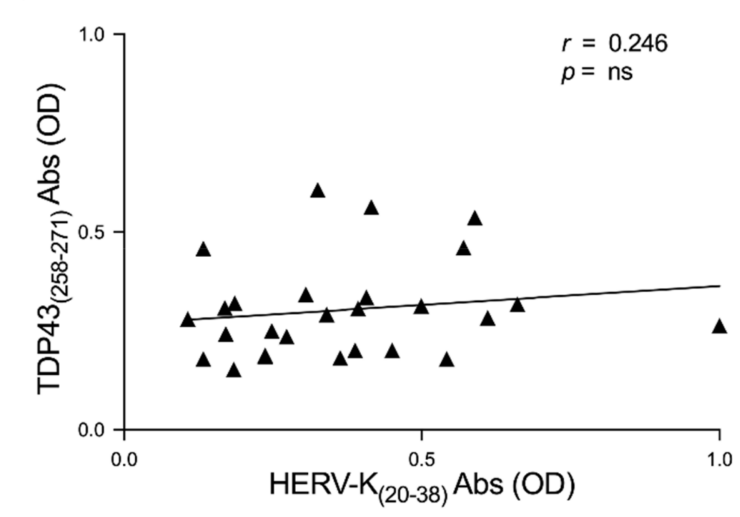

C

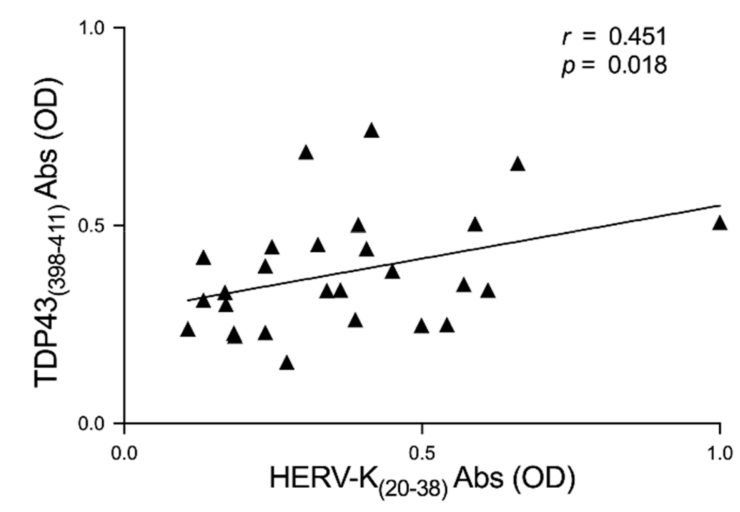

$E$

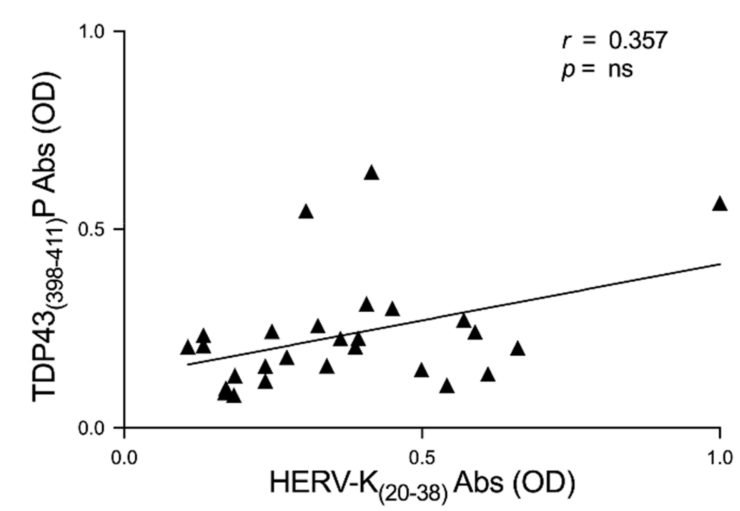

B

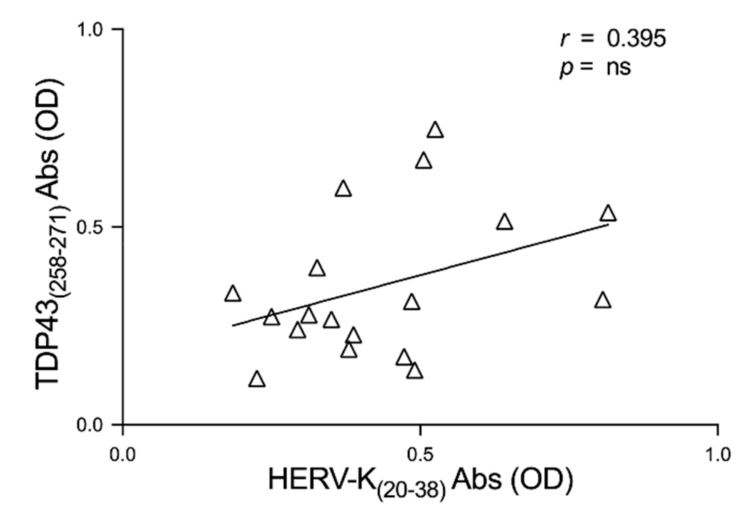

$\mathrm{D}$

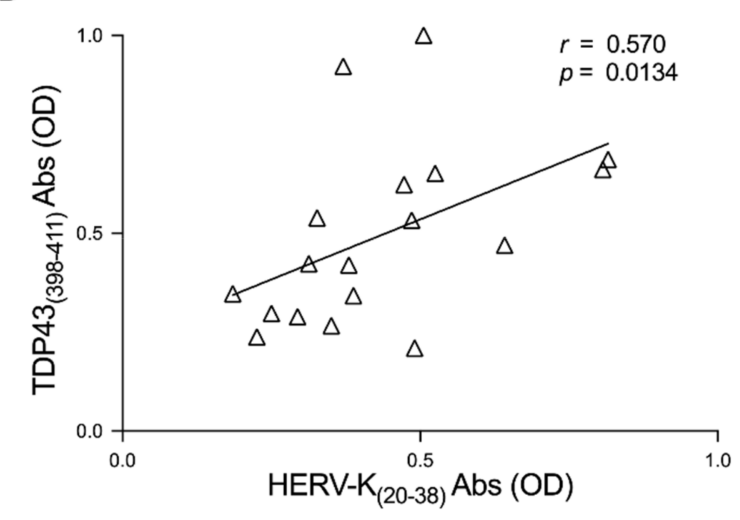

$\mathrm{F}$

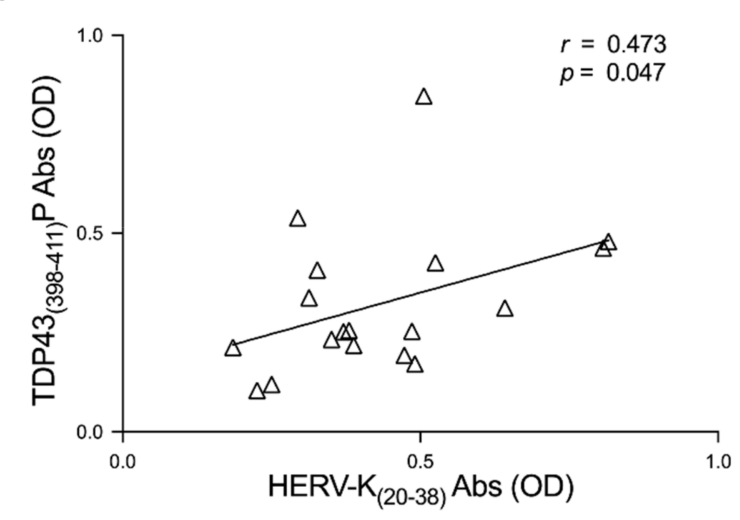

Figure 4. Scatter plots of antibodies to TDP-43-derived peptides and HERV-K env epitope in ALS populations. The graphs show the correlation between HERV-K-env-su $(20-38)$ and TDP-43(258-271), TDP-43 ${ }_{(398-411)}$, and TDP-43(398-411) $\mathrm{P}$ in ALS-ND patients $(\mathbf{A}, \mathbf{C}, \mathbf{E})$ and in ALS-LS (B,D,F).

\section{Discussion}

In this study, we investigated the humoral response directed against specific epitopes of the HERV-K envelope and TDP-43 proteins in human ALS plasma samples. Autoantibodies were found to be significantly higher in ALS patients compared with healthy control samples. These results confirm our previous data and highlight the reactivation of the human endogenous retrovirus in ALS pathology [14]. 
Likewise, Conti et al., documented increased levels of both anti-TDP-43 Abs and TDP-43 protein in ALS-serum patients compared with healthy controls, motor neuron disease mimics, and Alzheimer's and frontotemporal lobar degeneration patients, but autoantibodies and protein serum levels failed to correlate [36]. However, recent work has documented a decreased level of anti-TDP-43 Abs in ALS plasma samples [37].

Several studies [38,39] suggest an association between TDP-43 plasma levels and the cerebral accumulation of the protein; however, there is no strong scientific evidence that corroborates this intriguing hypothesis, and further studies are required.

The hypothesis regarding the possible physiological role of autoantibodies in the prevention of excessive accumulation of neuronal inclusions is in agreement with the data reported [40].

Otherwise, the literature has quite clearly proven that the cytoplasmic aggregation of phosphorylated, ubiquitinated, and truncated TDP-43 represents a pathologic hallmark across the clinical spectrum of ALS [6]. The C-terminal region of TDP-43, in particular, is a complex domain, which is essential in the mediation of liquid-liquid phase transitions in the protein, which is central for the biogenesis of various membrane-less organelles such as stress granules, even if makes them prone to misfolding and aggregation [41]. Cterminal fragments (CTFs) are found in cytoplasmic aggregates along with the full-length TDP-43 [6]. Regarding this, our results highlight the increased humoral response directed against selected C-terminal epitopes, including phosphorylated ones, of TDP-43 in ALS patients when compared with HCs (Figure 1). Associating the plasma antibody response with the cerebral accumulation of TDP-43 is particularly intriguing to have an overview of the cerebral accumulation of protein; nevertheless, further investigations are necessary to gain more insight into this aspect.

In order to investigate the role of autoantibodies as a novel biomarker in ALS, we searched for the presence of different autoantibodies in both ALS-ND and ALS-LS patients (Figure 2). The humoral response against the four epitopes selected did not exhibit any statistical difference, indicating that the Abs searched may be present in ALS individuals from the first months after the diagnosis. It would be very interesting to investigate the humoral response in a prospective study looking for autoantibodies at the first symptoms before a definite ALS diagnosis is made.

Different studies have documented the implication of HERV-K in ALS. An altered expression of HERV-K pol transcripts in post-mortem brain samples of ALS patients has been confirmed compared to patients with other nervous system diseases and healthy subjects [12]. HERV-K env transgenic cells and mice showed ALS-like pathological and neurological manifestations. Significantly increased levels of anti-HERV-K-env-su Abs were observed by Arru et al., in the serum and cerebrospinal fluid of ALS patients, hinting at the development of a humoral immune response to HERV-K in patients [14].

Furthermore, HERV-K pol-gene expression was found to correlate with TDP-43 mRNA in post-mortem brain tissue from patients with ALS [12], and we found a correlation between the humoral response against HERV-K-env-su $\mathrm{s}_{(20-38)}$ and TDP-43 epitopes.

Interestingly, no correlations have arisen in HCs. This endorses the results obtained in ALS patients and the specific relationship between HERV-K and TDP-43 in pathological conditions (Figure 3). Additional analysis, associated with the duration of the disease, has highlighted a slightly stronger correlation in LS-ALS compared to ND-ALS regarding the $\mathrm{Ab}$ levels between TDP-43 and HERV-K (Figure 4). If further studies confirm this observation, that relationship might be considered as a possible marker of ALS progression. With regard to this connection, it would be useful to conduct a prospective study and monitor the patients in ALS evolution.

Currently, the regulatory role of TDP-43 in the HERV-K expression is well known [13], but further and more in-depth studies are needed to understand whether a correlation between the humoral response against TDP-43 and HERV-K exists. 


\section{Conclusions}

Our investigation concludes that the epitopes of TDP-43 and HERV-K envelope identified by the Immune Epitope Database (IEDB) are highly immunogenic and recognized by the humoral response found in ALS patients in comparison with the weak response of the healthy control subjects analyzed. We are currently enrolling for a larger study to deepen our hypothesis. In particular, clinical data regarding ALS are going to be collected, in order to point out any putative relationship. It would be interesting to conduct a prospective study to better understand if our results are the consequences of the pathology, or if they are the result of a connection that might exist between TDP-43 and HERV-K env.

Currently, no specific biomarkers are available for the differential diagnosis of ALS, thus diagnostic and prognostic biomarkers for ALS remain a major unmet clinical need, and the results obtained in this work suggest a need for further study in this direction with a larger number of patients and controls.

Author Contributions: Conceptualization, L.A.S.; data curation and formal analysis, E.R.S. and L.A.S.; funding acquisition, L.A.S.; investigation, E.R.S. and L.A.S.; methodology, E.R.S.; project administration, L.A.S.; resources, L.A.S.; supervision, L.A.S.; samples acquisition and validation, G.A., I.R.Z. and P.S.; writing—original draft, E.R.S.; writing—review and editing, L.A.S. All authors have read and agreed to the published version of the manuscript.

Funding: This work was supported by the grant of Fondazione di Sardegna 2017 to L.A.S. UNISS FAR 2019, 2020 to L.A.S and PhD School of the University of Sassari to ERS.

Institutional Review Board Statement: Ethical approval was obtained from ASL 2324/CE 2016.

Informed Consent Statement: Informed consent was obtained from all subjects involved in the study.

Acknowledgments: All authors would like to thank all patients and their respective relatives for their collaboration in this study, Demetrio Vidili and AISLA, and Vanna Chessa, responsible for ventilated patient's ambulation, and all nurses for their collaboration in recruiting ALS patients.

Conflicts of Interest: The authors declare no conflict of interest.

\section{References}

1. Rowland, L.P.; Shneider, N.A. Medical Progress. Amyotrophic Lateral Sclerosis. N. Engl. J. Med. 2001, 344, 1688-1700.

2. Kiernan, M.C.; Vucic, S.; Cheah, B.C.; Turner, M.R.; Eisen, A.; Hardiman, O.; Burrell, J.R.; Zoing, M.C. Amyotrophic lateral sclerosis. Lancet 2011, 377, 942-955.

3. Logroscino, G.; Traynor, B.J.; Hardiman, O.; Chio, A.; Mitchell, D.; Swingler, R.J.; Millul, A.; Benn, E.; Beghi, E.; Eurals, F. Incidence of amyotrophic lateral sclerosis in Europe. J. Neurol. Neurosurg. Psychiatry 2009, 81, 385-390. [CrossRef]

4. Gordon, P.H. Amyotrophic Lateral Sclerosis: An update for 2013 Clinical Features, Pathophysiology, Management and Therapeutic Trials. Aging Dis. 2013, 4, 295-310. [CrossRef]

5. Martin, S.; Al Khleifat, A.; Al-Chalabi, A. What causes amyotrophic lateral sclerosis? F1000Research 2017, 6, 371. [CrossRef]

6. Neumann, M.; Sampathu, D.M.; Kwong, L.K.; Truax, A.C.; Micsenyi, M.C.; Chou, T.T.; Bruce, J.; Schuck, T.; Grossman, M.; Clark, C.M.; et al. Ubiquitinated TDP-43 in Frontotemporal Lobar Degeneration and Amyotrophic Lateral Sclerosis. Science 2006, 314, 130-133. [CrossRef]

7. Alonso, A.; Logroscino, G.; Hernán, M. Smoking and the risk of amyotrophic lateral sclerosis: A systematic review and meta-analysis. J. Neurol. Neurosurg. Psychiatry 2010, 81, 1249-1252. [CrossRef]

8. Scarmeas, N.; Shih, T.; Stern, Y.; Ottman, R.; Rowland, L.P. Premorbid weight, body mass, and varsity athletics in ALS. Neurology 2002, 59, 773-775. [CrossRef]

9. Bozzoni, V.; Pansarasa, O.; Diamanti, L.; Nosari, G.; Cereda, C.; Ceroni, M. Amyotrophic lateral sclerosis and environmental factors. Funct. Neurol. 2016, 31, 7.

10. Capozzella, A.; Sacco, C.; Chighine, A.; Loreti, B.; Scala, B.; Casale, T.; Sinibaldi, F.; Tomei, G.; Giubilati, R.; Tomei, F.; et al. Work related etiology of amyotrophic lateral sclerosis (ALS): A meta-analysis. Ann. Ig. Med. Prev. Comunita 2014, 26, 456-472.

11. Hughes, J.F.; Coffin, J.M. A Novel Endogenous Retrovirus-Related Element in the Human Genome Resembles a DNA Transposon: Evidence for an Evolutionary Link? Genomics 2002, 80, 453-455. [CrossRef]

12. Douville, R.; Liu, J.; Rothstein, J.; Nath, A. Identification of active loci of a human endogenous retrovirus in neurons of patients with amyotrophic lateral sclerosis. Ann. Neurol. 2010, 69, 141-151. [CrossRef] 
13. Li, W.; Lee, M.-H.; Henderson, L.; Tyagi, R.; Bachani, M.; Steiner, J.; Campanac, E.; Hoffman, D.A.; von Geldern, G.; Johnson, K.; et al. Human endogenous retrovirus-K contributes to motor neuron disease. Sci. Transl. Med. 2015, 7, 307. [CrossRef]

14. Arru, G.; Mameli, G.; Deiana, G.A.; Rassu, A.L.; Piredda, R.; Sechi, E.; Caggiu, E.; Bo, M.; Nako, E.; Urso, D.; et al. Humoral immunity response to human endogenous retroviruses $\mathrm{K} / \mathrm{W}$ differentiates between amyotrophic lateral sclerosis and other neurological diseases. Eur. J. Neurol. 2018, 25, 1076-e84. [CrossRef]

15. Manghera, M.; Ferguson-Parry, J.; Douville, R.N. TDP-43 regulates endogenous retrovirus-K viral protein accumulation. Neurobiol. Dis. 2016, 94, 226-236. [CrossRef]

16. Douville, R.N.; Nath, A. Human Endogenous Retrovirus-K and TDP-43 Expression Bridges ALS and HIV Neuropathology. Front. Microbiol. 2017, 8, 1986. [CrossRef]

17. Prasad, A.; Bharathi, V.; Sivalingam, V.; Girdhar, A.; Patel, B.K. Molecular Mechanisms of TDP-43 Misfolding and Pathology in Amyotrophic Lateral Sclerosis. Front. Mol. Neurosci. 2019, 12, 25. [CrossRef]

18. Buratti, E.; Baralle, F.E. Characterization and Functional Implications of the RNA Binding Properties of Nuclear Factor TDP-43, a Novel Splicing Regulator ofCFTR Exon 9. J. Biol. Chem. 2001, 276, 36337-36343. [CrossRef]

19. Hanson, K.A.; Kim, S.H.; Tibbetts, R.S. RNA-binding proteins in neurodegenerative disease: TDP-43 and beyond. Wiley Interdiscip. Rev. RNA 2011, 3, 265-285. [CrossRef]

20. Costessi, L.; Porro, F.; Iaconcig, A.; Muro, A.F. TDP-43 regulates $\beta$-adducin(Add2) transcript stability. RNA Biol. 2014, 11, 1280-1290. [CrossRef]

21. McDonald, K.K.; Aulas, A.; Destroismaisons, L.; Pickles, S.; Beleac, E.; Camu, W.; Rouleau, G.A.; Velde, C.V. TAR DNA-binding protein 43 (TDP-43) regulates stress granule dynamics via differential regulation of G3BP and TIA-1. Hum. Mol. Genet. 2011, 20, 1400-1410. [CrossRef]

22. Freibaum, B.D.; Chitta, R.K.; High, A.A.; Taylor, J.P. Global Analysis of TDP-43 Interacting Proteins Reveals Strong Association with RNA Splicing and Translation Machinery. J. Proteome Res. 2010, 9, 1104-1120. [CrossRef]

23. Huang, C.; Xia, P.Y.; Zhou, H. Sustained Expression of TDP-43 and FUS in Motor Neurons in Rodent's Lifetime. Int. J. Biol. Sci. 2010, 6, 396-406. [CrossRef]

24. Sephton, C.F.; Good, S.K.; Atkin, S.; Dewey, C.M.; Mayer, P.; Herz, J.; Yu, G. TDP-43 Is a Developmentally Regulated Protein Essential for Early Embryonic Development. J. Biol. Chem. 2010, 285, 6826-6834. [CrossRef]

25. Winton, M.J.; Igaz, L.M.; Wong, M.M.; Kwong, L.K.; Trojanowski, J.Q.; Lee, V.M.-Y. Disturbance of Nuclear and Cytoplasmic TAR DNA-binding Protein (TDP-43) Induces Disease-like Redistribution, Sequestration, and Aggregate Formation. J. Biol. Chem. 2008, 283, 13302-13309. [CrossRef]

26. Ayala, Y.M.; Zago, P.; D'Ambrogio, A.; Xu, Y.-F.; Petrucelli, L.; Buratti, E.; Baralle, F.E. Structural determinants of the cellular localization and shuttling of TDP-43. J. Cell Sci. 2008, 121, 3778-3785. [CrossRef]

27. Kametani, F.; Obi, T.; Shishido, T.; Akatsu, H.; Murayama, S.; Saito, Y.; Yoshida, M.; Hasegawa, M. Mass spectrometric analysis of accumulated TDP-43 in amyotrophic lateral sclerosis brains. Sci. Rep. 2016, 6, 23281. [CrossRef]

28. Buratti, E. TDP-43 post-translational modifications in health and disease. Expert Opin. Ther. Targets 2018, 22, 279-293. [CrossRef]

29. Hasegawa, M.; Arai, T.; Nonaka, T.; Kametani, F.; Yoshida, M.; Hashizume, Y.; Beach, T.G.; Buratti, E.; Baralle, F.; Morita, M.; et al. Phosphorylated TDP-43 in frontotemporal lobar degeneration and amyotrophic lateral sclerosis. Ann. Neurol. 2008, 64, 60-70. [CrossRef]

30. Neumann, M.; Kwong, L.K.; Lee, E.B.; Kremmer, E.; Flatley, A.; Xu, Y.; Forman, M.S.; Troost, D.; Kretzschmar, H.A.; Trojanowski, J.Q.; et al. Phosphorylation of S409/410 of TDP-43 is a consistent feature in all sporadic and familial forms of TDP-43 proteinopathies. Acta Neuropathol. 2009, 117, 137-149. [CrossRef]

31. Inukai, Y.; Nonaka, T.; Arai, T.; Yoshida, M.; Hashizume, Y.; Beach, T.G.; Buratti, E.; Baralle, F.E.; Akiyama, H.; Hisanaga, S.-I.; et al. Abnormal phosphorylation of Ser409/410 of TDP-43 in FTLD-U and ALS. FEBS Lett. 2008, 582, 2899-2904. [CrossRef]

32. Gu, J.; Wang, W.; Miao, S.; Chen, F.; Wu, F.; Hu, W.; Iqbal, K.; Gong, C.-X.; Liu, F. Protein Phosphatase 1 dephosphorylates TDP-43 and suppresses its function in tau exon 10 inclusion. FEBS Lett. 2018, 592, 402-410. [CrossRef]

33. Kametani, F.; Nonaka, T.; Suzuki, T.; Arai, T.; Dohmae, N.; Akiyama, H.; Hasegawa, M. Identification of casein kinase-1 phosphorylation sites on TDP-43. Biochem. Biophys. Res. Commun. 2009, 382, 405-409. [CrossRef]

34. Goh, C.W.; Lee, I.C.J.; Sundaram, J.R.; George, S.E.; Yusoff, P.; Brush, M.H.; Sze, S.K.; Shenolikar, S. Chronic oxidative stress promotes GADD34-mediated phosphorylation of the TAR DNA-binding protein TDP-43, a modification linked to neurodegeneration. J. Biol. Chem. 2018, 293, 163-176. [CrossRef]

35. Radunović, A.; Mitsumoto, H.; Leigh, P.N. Clinical care of patients with amyotrophic lateral sclerosis. Lancet Neurol. 2007, 6, 913-925. [CrossRef]

36. Conti, E.; Sala, G.; Diamanti, S.; Casati, M.; Lunetta, C.; Gerardi, F.; Tarlarini, C.; Mosca, L.; Riva, N.; Falzone, Y.; et al. Serum naturally occurring anti-TDP-43 auto-antibodies are increased in amyotrophic lateral sclerosis. Sci. Rep. 2021, 11, 1-6. [CrossRef]

37. Nielsen, A.K.; Folke, J.; Owczarek, S.; Svenstrup, K.; Winge, K.; Pakkenberg, B.; Aznar, S.; Brudek, T. TDP-43-specific Autoantibody Decline in Patients with Amyotrophic Lateral Sclerosis. Neurol. Neuroimmunol. Neuroinflammation 2021, 8, e937. [CrossRef] 
38. Verstraete, E.; Kuiperij, B.; van Blitterswijk, M.; Veldink, J.H.; Schelhaas, H.J.; Berg, L.H.V.D.; Verbeek, M.M. TDP-43 plasma levels are higher in amyotrophic lateral sclerosis. Amyotroph. Lateral Scler. 2012, 13, 446-451. [CrossRef]

39. Foulds, P.; McAuley, E.; Gibbons, L.; Davidson, Y.; Pickering-Brown, S.M.; Neary, D.; Snowden, J.S.; Allsop, D.; Mann, D.M.A. TDP-43 protein in plasma may index TDP-43 brain pathology in Alzheimer's disease and frontotemporal lobar degeneration. Acta Neuropathol. 2008, 116, 141-146. [CrossRef]

40. Zhou, Q.; Lehmer, C.; Michaelsen, M.; Mori, K.; Alterauge, D.; Baumjohann, D.; Schludi, M.H.; Greiling, J.; Farny, D.; Flatley, A.; et al. Antibodies inhibit transmission and aggregation of C9orf72 poly- GA dipeptide repeat proteins. EMBO Mol. Med. 2017, 9, 687-702. [CrossRef]

41. Harrison, A.F.; Shorter, J. RNA-binding proteins with prion-like domains in health and disease. Biochem. J. 2017, 474, 1417-1438. [CrossRef] 